ISSN 1991- 8690

Website: http://jsci.utq.edu.iq
الترقيم الدولي • 1991 - 1979

Email: utjsci@utq.edu.iq

\title{
Electronic excitation produced by proton moving inside polystyrene using Drude model
}

\author{
Sahar Mezher Mutashar \\ Department of Medical physics-College of Medicine- Thi Qar University \\ Emil: saheralasdi@yahoo.com
}

\section{$\underline{\text { Abstract }}$}

The electronic response of target is characterized by its energy-loss function (ELF), which it described by several models that fit the available experimental data (at zero momentum transfer), but use different approaches to obtain the Bethe surface so that is to extend the (ELF) to include any energy and momentum transferred. In the present research the Drude dielectric formalism had been used to calculate the probability for an energetic proton to produce electronic excitations in target, Target material used in this research is a polymeric material which is polystyrene. And calculate the effects of ionization fraction of Partial Stopping Power Effective Charge (PSPEC) of polystyrene have been studied and taking into consideration the electronic excitation in the target. Drude Energy Loss Function DELF has been used to take in the consideration the charge exchange, screening length and charge density. FORTRAN-90 program $H$ - Polystyrene.for has been written for the mathematical equations using software Compaq visual Fortran90 V 6.6, for linking and execute.

Key words: polystyrene, energy loss function, excitation energy.

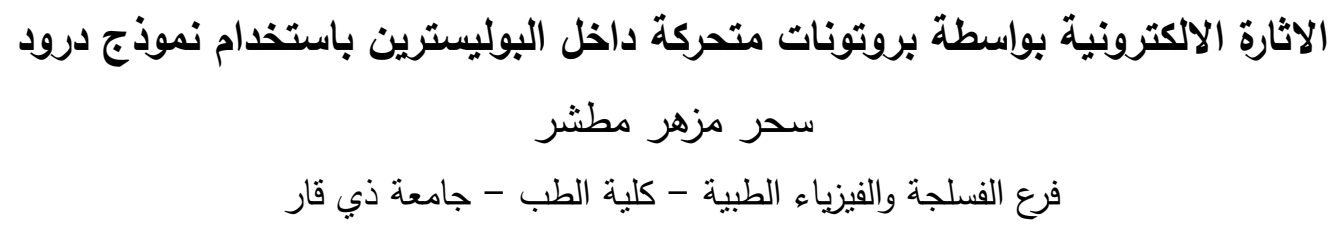

$\underline{\text { الخلاصة }}$

تتميز الاستجابة الإكترونية للهذف بدالة فقدان الطاقة (ELF) والني وصفها العديد من النماذج التي تتاسب البيانات التجريبية المتاحة (عند الزخم

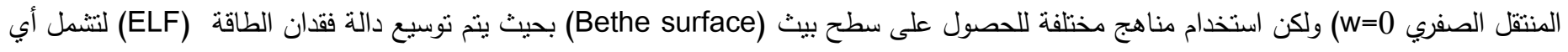

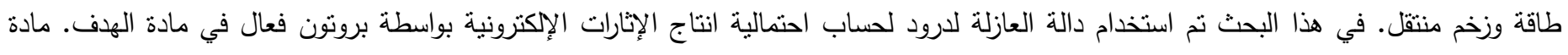
الهدف المستخدمة في هذا البحث هي مادة بوليمرية وهي البوليسترين • وقد نم دراسة ناثير الكسر الايوني للشحنة المؤثرة لطاقة الايقاف الجزيئي من البوليسترين ومع الأخذ بعين الاعتبار الإثارة الإكترونية في الهدف. وقد اخذت دالة فقدان الطاقة لدرود DELF (PSEC)

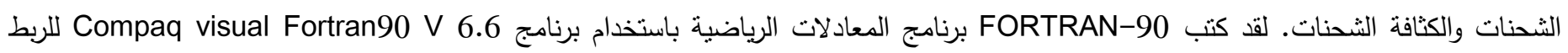

\section{Introduction}

Polystyrene the molecular formula is $C_{8} H_{8}$. It is a multipurpose polymer that is used in varied applications in the rigid and foamed form. General purpose polystyrene is clear and hard which is used in packaging, laboratory ware, and electronics. The excellent physical and processing properties make polystyrene suitable for a lot of applications than any other plastic. Its impact on the environment of everyday materials is increasingly important if we are to reduce our carbon footprint and protect our natural heritage for future generation [1]. On the other hand, the study of energy deposition a activations is a problem of concern to the core and applied research in many areas ,such a scion 
implantation, ion beam analysis and modification of materials, and radiation image, radiation therapy or space research[2]. Therefore, given the importance of the polystyrene, it is recommend inaccurately stopping power in a wide range of knowledge range of ion energies, because it plays a key role in the ion beam Analysis experiments. The knowledge of the features of the transmission and absorption of low, intermediate and high energy protons in elemental materials is of great importance for the experimental methods in nuclear and atomic physics. It is also useful in understanding the interaction of particles with matter. The heavy charged particles like protons interact with matter primarily through coulomb forces between a positive and negative charge of the orbital electrons within the absorber atoms. Thus the present work performed a study on the stopping power of proton in different materials [3]. Projectile excitation / ionization contributes significantly to stopping when the number of electrons carried by the projectile is comparable to or greater than that of the target, i.e., In the velocity range of heavy screening where stopping by target excitation / ionization is mostly due to outer electrons. The process can become dominant when the number of weakly-bound projectile electrons exceeds the number of weakly-bound target electrons, i.e., For nearneutral ions with $Z_{1}>Z_{2}$ at velocity $v \leq v_{0} Z_{1}^{2 / 3}$ here, $Z_{1}$ and $Z_{2}$ denote the atomic numbers of the projectile and target material conclude that the projectile excitation/ionization may constitute a significant and even dominate contribution to the stopping power for a heavy ion, effectible molecular effects is generally small [4]. Therefore, the relative significance of coherent stopping by target excitation or ionization will get diminished by an increasing contribution to stopping by projectile excitation or ionization [5].

\section{Theoreticalbackground}

\section{1- Description of the Target ELF}

The key parameter to obtain reliable results for the energy losses is the energy-loss function (ELF) of the material, $\operatorname{Im}[-1 / \epsilon(\mathrm{k}, \omega)]$, since it contains all the information about the electron excitation spectrum of the target. Thus, it is essential to use a good description of the target ELF for the whole k- $\omega$ plane (that is, the Bethe surface) [6]. The wellknown Lindhard dielectric function is applicable to only a limited number of so-called nearly-free-electron materials, like aluminum. For other targets, a commonly used method for obtaining the ELF is to employ the experimental energyloss function at $\mathrm{k}=0$ (optical limit) and extend it to $\mathrm{k}>0$ by introducing suitable dispersion relationship schemes [7]. Experimental information about the ELF at $\mathrm{k}=0$ can be obtained for a number of materials, including polystyrene from the measurements of optical magnitudes[8]. However, experimental information about the ELF at $\mathrm{k} \neq 0$ is limited. For this reason, it is necessary to model the evolution of the optical ELF with finite $\mathrm{k}$ in order to calculate magnitudes such as $\mathrm{P}$ and $\langle\mathrm{E}\rangle$. This approach allows the separation of contributions from outer electron and the inner-shell electron excitations to the target ELF. The excitation of the outer electrons of the solid, including both collective and singleparticle excitations, are described by fitting the experimental optical ELF $(k=0)$ [8],

$$
\operatorname{Im}\left[-\frac{1}{\varepsilon(k, \omega)}\right]_{\text {outer }}=\operatorname{Im}\left[-\frac{1}{\varepsilon(k=0, \omega)}\right]_{\exp }
$$

The Plasmon Pole Approximation (PPA) is applicable for a projectile of high velocity compared to the Fermi velocity, to give a good approximation to the Lindhard dielectric function. It accounts for the collective electron-gas behavior at small and the free-particle behavior at large, are included [9]

$$
\in(\overrightarrow{\mathrm{k}}, \omega)=1+\frac{\omega_{p}^{2}}{\omega_{g}^{2}+\beta^{2} k^{2}+\frac{k^{4}}{4}-\omega(\omega+i \gamma)}
$$

Where $\beta^{2}=3 \mathrm{v}_{\mathrm{F}}^{2} / 5$ is the square of the mean propagation velocity of disturbances in the system. A both collective and single-particle effect in an electron gas are represented in and $\omega_{p}$ is the Plasmon frequency 
$\omega_{p}=3^{1 / 2} / r_{s}^{3 / 2}$ and the effective band gap energy $\omega_{g}$ semiconductors and insulators give a collective resonance frequency $\Omega_{o}=\left(\omega_{p}^{2}+\omega_{g}^{2}\right)^{1 / 2}[10]$.

The form of plasmon-pole dielectric functions from Drude dielectric function [6],

$$
\operatorname{Im}=\left[\frac{-1}{\varepsilon(k, \omega)}\right]_{\text {outer }}=\sum_{i} \frac{A_{D i} \gamma_{i} \omega}{\left[\left[\omega_{i}(k)\right]^{2}-\omega^{2}\right]^{2}+\left(\gamma_{i} \omega\right)^{2}} \theta\left(\omega-\omega_{\text {th }, i}\right)
$$

Where $A_{D i}, \omega_{i}, \gamma_{i}$ and $\omega_{t h, i}$ are the intensity, position, width and threshold respectively of Drude-ELF peaks, $\theta(. .$. represent the Heaviside step function [11].

\section{Partial Stopping Power Effective Charge (PSPEC)}

Consider an atomic projectile moving with velocity $v$ induce medium. Let the medium be the valence electrons of a solid of density $\left(\frac{1}{\frac{4}{3} \pi r_{s}^{3}}\right)$, where $r_{S}$ in the radius of the average volume occupied by each electron in unit of Bohr radius $a_{0}=\hbar^{2} / m e^{2}=0.529 A^{0}$,Two things had [12]:

(i) The onrushing electrons eject bound electrons for the projectile. This leaves the projectile with an ionic charge $\mathrm{Q}=\left(Z_{1}-N_{e}\right)$ e, where $N_{e}$ is the number of electrons still bound to projectile. The degree of ionization $q=\left(Z_{1}-N_{e}\right) / Z_{1}$ is $v$ dependent.

(ii) The projectile through Coulomb interaction transfers momentum to electrons of the medium. This momentum transfer $2 Z_{1}^{*} e^{2} / b_{0} v_{1}$ is proportions to an effective charge $Z_{1}^{*}$.

The energy loss of the projectile $\sim 2 Z_{1}^{* 2} e^{4} / m v_{1}^{2} b_{0}^{2}$, when integrated over electrons with all possible impact parameter ( $b_{0}$ Collision diameter) yields the rate of energy loss of the ion in the medium. That is, the stopping cross section $\left(S=\frac{1}{N} \cdot \frac{d E}{d x}\right)$ of the medium for an ion is proportions with square of effective charge $Z_{1}^{* 2}$. [13].

Brandt and Kitagawa (1982) have derived a formula for Partial Stopping Power Effective Charge (PSPEC) fraction for projectile ionization $q_{e}$ in a variation of statistical approximation.

The Stopping of a single charged projectile is given as[14]:

$$
S=-\frac{1}{N} \frac{d E}{d x}=\frac{2}{\pi v^{2}} \int_{0}^{\infty} \frac{d k}{k}|\rho(k)|^{2} \int_{0}^{k v} \omega d \omega \operatorname{Im}\left[\frac{-1}{\varepsilon(k, \omega)}\right],
$$

The formula of $\rho(k)$ is given as follows,

$$
\rho(k)=Z_{1} \frac{q+(k \Lambda)^{2}}{1+(k \Lambda)^{2}} r_{0}
$$

Where $\Lambda$ is the screening length given by Brandt and Kitagawa (1982) [13]

$$
\Lambda=\frac{2 r(1-q)^{2 / 3}}{Z_{1}^{1 / 3}[1-(1-q) / 7]} r_{0}
$$

Where r=0.240a.u, $r_{0}=1 a . u=0.529 A^{0}$ is Bohr's radius and $q=1-\left(N_{e} / Z_{1}\right)$ is the charge exchange. 
The PSPEC fraction is given as [15]:

$\zeta\left(\mathbf{q}_{(\mathbf{r})}\right)=\left[\frac{s_{\mathrm{q}_{(\mathrm{r})}}}{s_{\mathrm{q}_{(\mathrm{r})=1}}}\right]^{1 / 2}$

Where $S_{(q=1)}$ is the Stopping Power for the bare nucleus of the projectile given in Eq. (4), which represents the Stopping Power of fully stripped projectile in matter.

\section{3- Bragg's additivity rule}

Bragg's rule [16] of linear additivity states that the electronic stopping cross-section $S^{A B}$ of atwo-component $\operatorname{target} A$ and $B$ is the weighted mean of the stopping cross -section $S^{A}$ and $S^{B}$ of its constituents $A$ and $B$,

$S^{A B}=C_{A} S^{A}+C_{B} S^{B}$

Where: $C_{A}$ and $C_{B}=1-C_{A}$ are the molar fractions of components $A$ and $B$, respectively.

The sum extends overall momentum transfers and all final states obviously, linear additivity can fail when $A$ and $B$ form a compound. Because of chemical binding, some of the values of energy transfer from projectile to the target for a collision process $(i)$ the corresponding cross-section at projectile energy $E_{i}$ can change, leading to well-known deviations from Bragg's rule for compounds [17].

Bragg's is possible to apply a rule to combine the ability to get the suspension of the compound and that the capacity of suspension of the constituent elements of the compound are expressed as base Bragg's to bring in terms of stopping power and mass as follows[18]:

$(S)_{\text {comp. }}=\sum_{i} w_{i}(s)_{i}$

Where: $(s)_{i}$ is the stopping power of the element (i) in the composite, and

$w_{i}=n_{i} A_{i} / A_{c}$

$\left(w_{i}\right)$ represents the percentage of presence of the element ( $\left.\mathrm{i}\right)$ in the composite (fraction by weight), $\left(A_{i}, A_{c}\right)$ are the mass number of the element (i) and composite.

Where: $A_{c}=\sum_{i} n_{i} A_{i}$

$S_{\text {bragg's }}=\frac{\sum_{i} W_{i}\langle Z / A\rangle_{i} S_{i}}{\langle Z / A\rangle_{\text {comp }}}$

$\langle\mathrm{Z} / \mathrm{A}\rangle_{\text {comp. }}=\sum_{i} w_{i}\left(\frac{Z}{A}\right)_{i}$

\section{4- The Probability and Average Energy of Electronic Excitation}

When a swift projectile with mass $M_{1}$, atomic number $Z_{1}$, kinetic energy $T$ and moves inside a solid, it induces electronic excitations in the material, losing energy in the process [18]. This energy loss mechanism is the dominant one. These electronic excitations can correspond to excitations or ionizations of individual electrons or even excitations of collective modes in the target electron gas.

The dielectric formalism [16] provides a way of studying the response of the electronic system of the target to the perturbation represented by the projectile. The key parameter of the problem is a correct description of the dielectric function of the material $\epsilon(k, \omega)$, which contains all the information about the electronic excitations that the material can sustain. Within this framework the probability per unit path length $P_{\mathbf{q}_{(\mathrm{r})}}\left(T_{k e}, E\right)$ that a projectile withcharge state $\left(\mathrm{q}_{(\mathrm{r})}\right)$ and energy $(T)$ produces in the target an excitation of energy $E_{e s}=\hbar \omega$ irrespective of its momentum, in atomic unit (a.u), $\hbar=1$, [8]is given by,

$P_{\mathbf{q}_{(\mathbf{r})}}(T, E)=\frac{m_{1} e^{2}}{\pi \hbar^{2} T} \int_{k_{\min }}^{\infty} \frac{d k}{k} \rho_{\mathbf{q}_{(\mathbf{r})}}^{2}(k) \operatorname{Im}\left[\frac{-1}{\epsilon(k, \omega)}\right]$

where:

$k_{\text {min }}=\omega / \sqrt{2 T_{k e} / m_{1}}$,

Present work, $\epsilon(k, \omega)$ is the dielectric function given in Eq. (1) has been utilized in the calculations in the interaction of Protons with polystyrene.Hence, the mean energy lost by the projectile per unit path length (the so called Stopping Power or Stopping Force) can be calculated by integrating over all possible energy transfer:

$\frac{d T}{d X}=\int_{0}^{\infty} \omega \cdot d \omega \cdot P_{\mathbf{q}_{(\mathbf{r})}}\left(T_{k e}, \omega\right)$ 
The mean energy of the electronic excitations $\left\langle E_{\mathrm{q}_{(\mathrm{r})}}(T)\right\rangle$ inducedby theprojectile can be written as[8]:

$\left\langle E_{\mathbf{q}_{(\mathbf{r})}}(T)\right\rangle=\frac{\int_{0}^{\infty} d E E P_{\mathbf{q}_{(\mathbf{r})}}(T, E)}{\int_{0}^{\infty} d E P_{\mathbf{q}_{(\mathbf{r})}}(T, E)}$

The charge state $\mathbf{q}_{(\mathbf{r})}$ of the projectile inside the target can varythrough capture and loss processes and depends on its energy $T$. However, when charge equilibrium is reached, the probability $\phi_{\mathbf{q}_{(\mathbf{r})}}(T)$ of finding the projectile in a charge state remains constant for each incident energy $T$ Put another way average over all possible charge states $\left(\mathbf{q}_{(\mathbf{r})}=0\right.$ and 1 for $H)$ in order to obtain the energy distribution, $P(T, E)$ and the mean energy, $\langle E(T)\rangle$ of the electronic excitations produced in the target as[18]:

$P(T, E)=\sum_{\mathbf{q}_{(\mathbf{r})}=0}^{1} \phi_{\mathbf{q}_{(\mathbf{r})}}(T) P_{\mathbf{q}_{(\mathbf{r})}}(T, E)$

$\left\langle E_{\mathbf{q}_{(\mathbf{r})}}(T)\right\rangle=\frac{\int_{0}^{\infty} d E E \sum_{\mathbf{q}_{(\mathbf{r})}=0}^{1} \emptyset_{q_{(r)}}(T) P_{\mathbf{q}_{(\mathbf{r})}}(T, E)}{\int_{0}^{\infty} d E \sum_{\mathbf{q}_{(\mathbf{r})}=0}^{1} \emptyset_{q_{(r)}}(T) P_{\mathbf{q}_{(\mathbf{r})}}(T, E)}$

\section{$\underline{\text { Results }}$}

In the present work, the energy loss function $\operatorname{Im}\left[\frac{-1}{\epsilon(k, \omega)}\right]$ at $\mathrm{k}=0$ based on eq.(2)is shown in fig (1). The maximum energy loss function of polystyrene is about 1.4 at energy transferred $20 \mathrm{eV}$ as shown in Fig. (1)

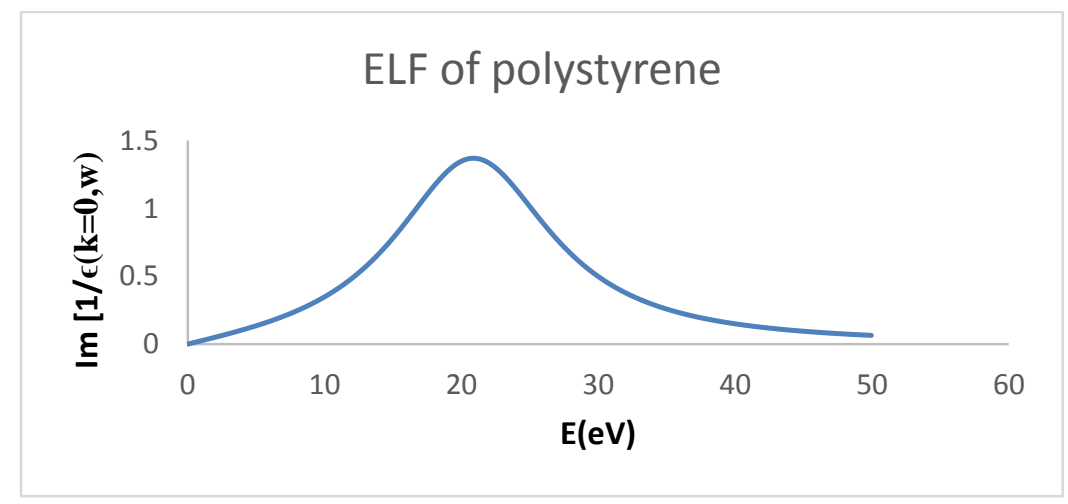

Fig.(1) shows the energy loss function of polystyrene in the optical limit $(\mathrm{k}=0)$ as a function of the transferred energy $\mathrm{E}$.

The calculation of the previous magnitudes requires the description of the projectile charge density through $\rho_{\mathbf{q}_{(\mathbf{r})}}(k)$ ofthe target excitation spectrum by means of its energy-loss function $(\boldsymbol{E} \boldsymbol{L} \boldsymbol{F}), \operatorname{Im}\left[\frac{-1}{\boldsymbol{\epsilon}(\boldsymbol{k}, \boldsymbol{\omega})}\right]$. The former accounts for with the model proposed by Brandt and Kitagawa [13] because it is reliable and provides analytical expressions for $\rho_{\mathbf{q}_{(\mathbf{r})}}(k)$.

Second, we calculated stopping power numerically for individual element given in polystyrene $C_{8} H_{8}$ applying Bragg's role :

Stopping Power for polystyrene $S_{p s}\left(\mathrm{q}_{(\mathrm{r})}\right)[17]$ :

$S_{p s}(q)=8 S_{C}\left(\mathrm{q}_{(\mathrm{r})}\right)+8 S_{H}\left(\mathrm{q}_{(\mathrm{r})}\right)$

Where: $S_{c}\left(\mathbf{q}_{(\mathbf{r})}\right), S_{H}\left(\mathbf{q}_{(\mathbf{r})}\right)$, are the Stopping Power of Carbon-C and Hydrogen-H, at charge fraction, $\mathrm{q}_{(\mathrm{r})}$.

The calculation of stopping power according to Brandt and Kitagawa $(\boldsymbol{B K})$ and Drude of the dielectric function as given in Eq. (4). The equations in present work had been solved numerically by writing a program in FORTRAN 90, and the result is shows in fig.(2) 


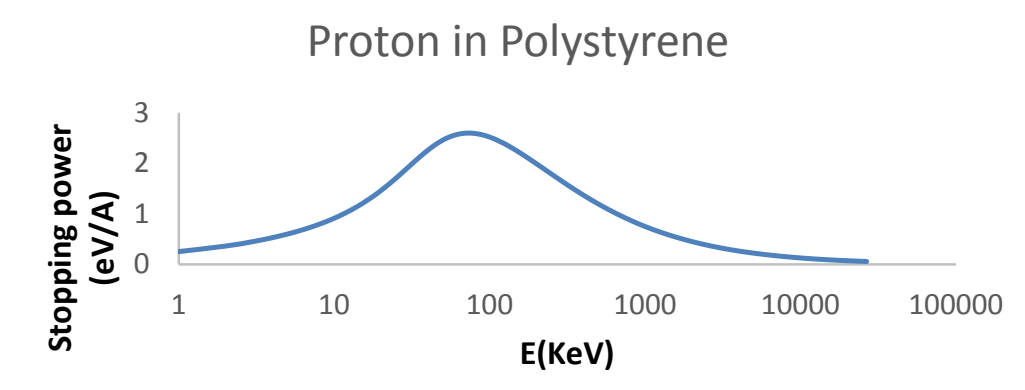

Fig.(2) show stopping power of polystyrene for beam of protons with energy $\mathrm{E}(\mathrm{keV})$

Then the (PSPEC) of polystyrene as given in Eq.(6) is calculated numerically, and it's variation with charge fraction $q$ for different incident proton energy $T(0.05,0.25,1,2,2.5 \mathrm{Mev} / \mathrm{u})$ is inversely proportional with Hydrogen incident energy, $\mathrm{T}$ and at $\mathrm{q}_{(\mathrm{r})}=1, \zeta\left(\mathrm{q}_{(\mathrm{r})}\right)=1$ as shown in fig.(3).

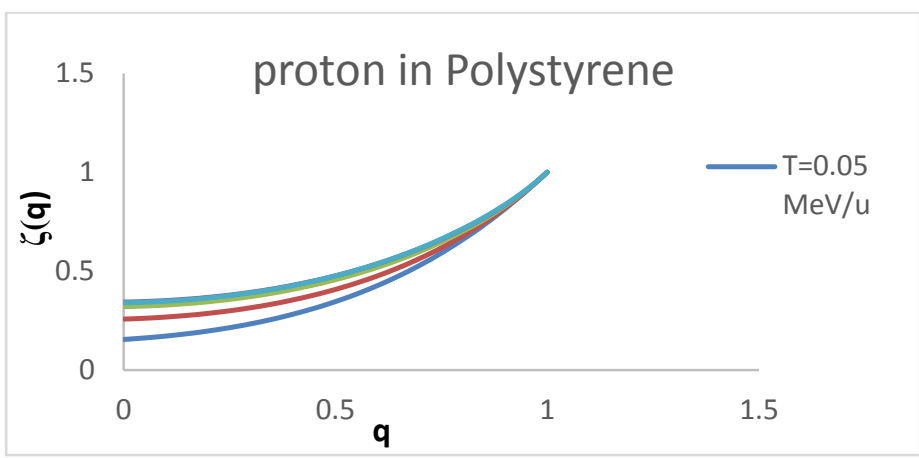

Fig.(3) PSPEC fraction $\zeta(q)$ for polystyrene using with ionization fraction, $\mathrm{q}_{(\mathrm{r})}$ for different incident proton energy $\mathrm{T}(0.05,0.25,1,2,2.5) \mathrm{Mev} / \mathrm{u}$.

The probability per unit path length, $\mathrm{P}(\mathrm{T}, \mathrm{E})$ based on Eq. (16), for the proton beam (having incident energies (T $=0.05 \mathrm{MeV}-2.5 \mathrm{MeV}$ ) of producing an electronic excitation of energy $\mathrm{E}$ in polystyrene is shown in Fig.(3) By using PPA has given in Eq. (2). The probability $\mathrm{P}$ decreases as the proton energy increases, indicating that a larger number of electronic excitations are produced when lower incident energies $\mathrm{T}$ are used. The values of P predicted by PPA agree at large $\mathrm{T}$, and this is very important because this range of $\mathrm{T}$ corresponds to the Bragg peak [6].

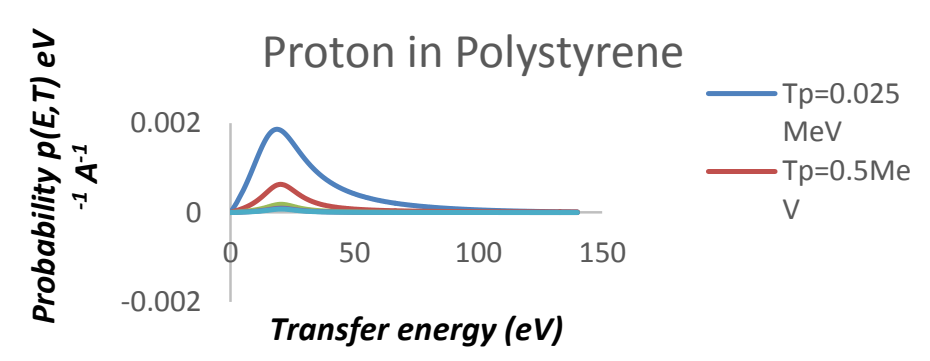

Fig. (4) Probability per unit path $\mathrm{P}(\mathrm{E}, \mathrm{T})$ that a proton projectile induces electronic excitation of energy $\mathrm{E}$ in polystyrene for different incident proton energy $\mathrm{T}(0.05,0.25,1,2,2.5) \mathrm{Mev} / \mathrm{u}$. 
The mean energy $\langle\mathrm{E}\rangle$ as given in Eq. (15), of the electronic excitations produced by a proton in polystyrene depicted is shown in Fig. (5). It is seen that $\langle E\rangle$ increases with the proton energy $T$,

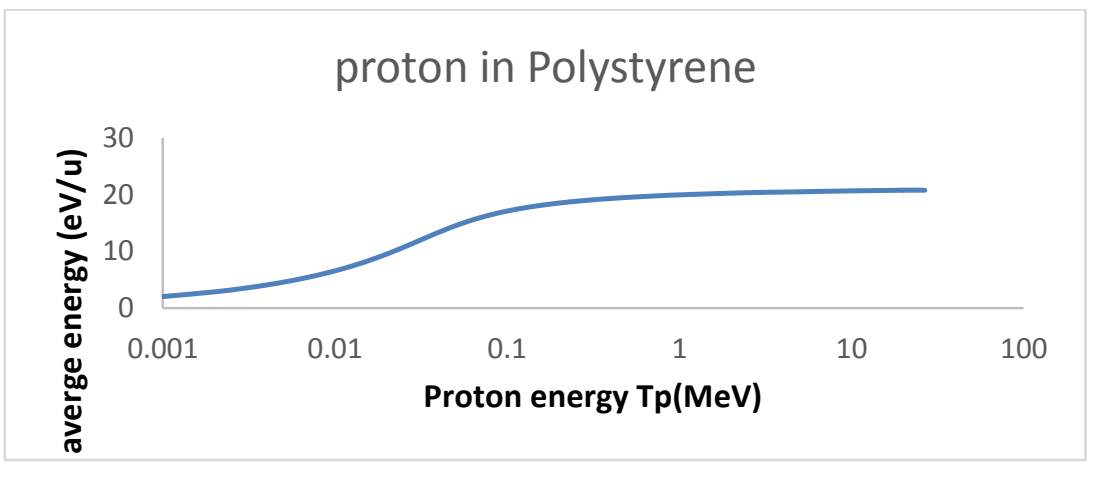

Fig.(5) Average Energy <E>for proton projectile induces in polystyrene

\section{Conclusions}

In the present work, stopping power, PSPEC fraction, the probability and the average energy of electronic excitation of the hydrogen ion beam in polystyrene target as been calculated using the Drude dielectric formalism. Results were obtained by executing a program $\mathrm{H}$-Polystyrene.for. The spectral distribution has been calculated of the electronic excitations induced by a proton in polystyrene. For this purpose, we use the dielectric formalism and obtain the target energy-loss function, I'm $[1 / \epsilon(\mathrm{w}, \mathrm{k})]$, which decrease with increasing in energy of the proton beam.Stopping power has the same behavior of ELF which decrease with increasing in proton's energy. A formula for the Partial Stopping Power Effective Charge (PSPEC) has been obtained based on the BK model with the dielectricfunction method and a modified screening length of the projectile. This formula provides the effective charge for the scaling of the collision energy loss with the BK effective-charge model. There is a shoulder for incident $\mathrm{H}$-ions with energy $T \approx 0.05 \mathrm{Mev} /$ you while this shoulder disappear at $T \geq 1.0 \mathrm{Mev} / \mathrm{you}$ and this is because of the low incidence $\mathrm{H}$-ion the time of interaction is large which cause a high Probability of interest. The values of $(\zeta)$ have a significant dependence on velocity at low charge state $\left(\mathrm{q}_{(\mathrm{r})}=0\right.$ ) While the dependence becomes less important at high charge state $\left(q_{(r)}>0\right)$. The spectral distribution of the electronic excitations induced by proton in polystyrene is calculated and found that, regardless of proton energy, the probability distribution $\mathrm{P}(\mathrm{E}, \mathrm{T})$, which is related to the number of electronic excitations of a given energy $\mathrm{E}$, decreases with the incident proton energy $\mathrm{T}$. On the other hand the mean energy $\langle\mathrm{E}\rangle$ of the electronic excitations increases monotonically with the proton energy $\mathrm{T}$.

\section{References}

1- P Meenakshi S E Noorjahan,R Rajini, U Venkateswarlu, C Rose and T P Sastry Bull,2002,. Mater. Sci., Vol.25, No.1, pp. 25-29.

2- P. Sigmund, I. S. Bitensky, and J. Jensen. ,1996 ,Nucl. Instrum. and Methods Phys. Res. B112,

3- Vibhuti Yadav,, S.N.L.Sirisha, Sonali Bhatnagar,2012,Proceedings of the DAE Symp. on Nucl. Phys. 57734

4- J. F. Ziegler, J. P. Biersack and M. D. Ziegler,2010, Nuclear Instruments and Methods in Physics Research Section B, Volume 268, Issue 11-12, p. 1818-1823.

5- Al-Gara'wi Mohammed Abdul Hamza Mohammed ,2005, Stopping of Molecules and Clusters in Solids, PhD thesis.

6- I. Abril, M. R. Garcia, C. D. Denton, I. Kyriakou and D. Emfietzoglou, 2011,"Energy loss of hydrogen and helium-Ion beams in DNA: Calculations based on a realistic energy-loss function of the target," Radiat. Res., vol. 175, pp. 247-255.

7- D. Emfietzoglou, I. Abril, R. Garcia-Molina, I. D. Petsalakis, H. Nikjoo, I. Kyriakou and A. Pathak, 2008,Nucl. Instrum. M ethods B., vol. 266, pp. 1154-1161. 
8- A. Isabel, D. Cristian, A. Denton, A. Pablo De Vera, K. Ioanna, E. Dimitris and G. M. Rafael, 2010," Nuclear Instruments and Methods in Physics Research B., vol. 268, pp. 1763-1767.

9- D. Pines, 1964, Elementary excitations in solids. New York: Benjamin.

10- Z. Cai and P. Coutier, 2005, J. Phys. Chem. B., vol. 109, pp. 4796-480.

11- P. M. Echenique, R. H. Ritchie and M. Brandt,1979, Phys. Rev. B., vol. 20, p. 2567.

12- W. Brandt and M. Kitagawa, 1982, Phys. Rev. B., vol. 25, p. 5631.

13- Brandt W. and Kitagawa M. 1982 , Phys. Rev. B25, 5631

14- LiM. M., D.J. O'Connor and H. Timmers , (2004), Nucl. Instr. And Meth .In Phys. Res. B222 11

15- Bethe H. , 1930, Ann. Physic, Vol.5, pp.325.

16- Lindhard J. K. Dan. Vidensk. Selsk, 1954, Mat. Fys. Medd. 28, 8.

17- Ziegler J. F. and J.M. Manoyan, 1988, Nucl. Instrum. Methods P 1763-1767hys. Res., Sect. B 35, 215

18- Awfa Zuhair Khudiar ,Khalid Abdul Wahab ,Riayhd K. A. Al-Ani ,2014,Journal of Asian Scientific Research,, 4(3): 139-148 